\title{
Fundamental Parameters of Four Massive Eclipsing Binaries in Westerlund 1
}

\author{
E. Koumpia and A. Z. Bonanos \\ National Observatory of Athens, Institute of Astronomy \& Astrophysics, \\ I. Metaxa \& Vas. Pavlou St., Palaia Penteli GR-15236 Athens, Greece \\ koumpia@astro.noa.gr, bonanos@astro.noa.gr
}

\begin{abstract}
We present fundamental parameters of four massive eclipsing binaries in the young massive cluster Westerlund 1 . The goal is to measure accurate masses and radii of their component stars, which provide much needed constraints for evolutionary models of massive stars. Accurate parameters can further be used to determine a dynamical lower limit for the magnetar progenitor and to obtain an independent distance to the cluster. Our results confirm and extend the evidence for a high mass for the progenitor of the magnetar.
\end{abstract}

Keywords. open clusters and associations: individual (Westerlund 1), stars: fundamental parameters, stars: early-type, binaries: eclipsing, stars: Wolf-Rayet

\section{Introduction}

Westerlund 1 (Wd1) is one of the most massive young clusters known in the Local Group of galaxies, with an age of 3-5 Myr. It was discovered by Westerlund (1961), but remained unstudied until recently due to the high interstellar extinction in its direction. It contains an assortment of rare evolved high-mass stars, such as blue, yellow and red supergiants, Wolf-Rayet stars, a luminous blue variable, many OB supergiants, as well as four massive eclipsing binary systems (Wddeb, Wd13, Wd36, WR77o, see Bonanos 2007). Furthermore, the magnetar CXO J164710.2-455216 was discovered in the cluster by Muno et al. (2006) in X-rays. This magnetar is a slow X-ray pulsar that is assumed to have formed from a massive progenitor star.

Eclipsing binaries provide the only accurate way for the measurement of masses and radii of stars. Thus, the study of these systems in the cluster is important for the following reasons: (1) the determination of fundamental parameters of the component stars (mass, radii, etc.), in order to increase the small sample of massive stars with well known physical parameters, (2) the test of stellar models for the formation and the evolution of massive stars, (3) the determination of a dynamical lower limit for the mass of the magnetar progenitor and (4) EBs present a great opportunity for an independent measurement of the distance, based on the expected absolute magnitude.

\section{The individual binaries}

We have analyzed spectra of all four eclipsing binaries, taken in 2007-2008 with the 6.5 meter Magellan telescope at Las Campanas Observatory, Chile. The spectra were reduced and extracted using IRAF. For the determination of the radial velocities, we adopted a $\chi^{2}$ minimization technique, which finds the least $\chi^{2}$ from the observed spectrum and fixed synthetic TLUSTY models (Lanz \& Hubeny 2003). For this purpose, we used the narrow Helium lines $(\lambda \lambda 6678,7065)$, as they are less sensitive to systematics, rather than the broader hydrogen lines. 

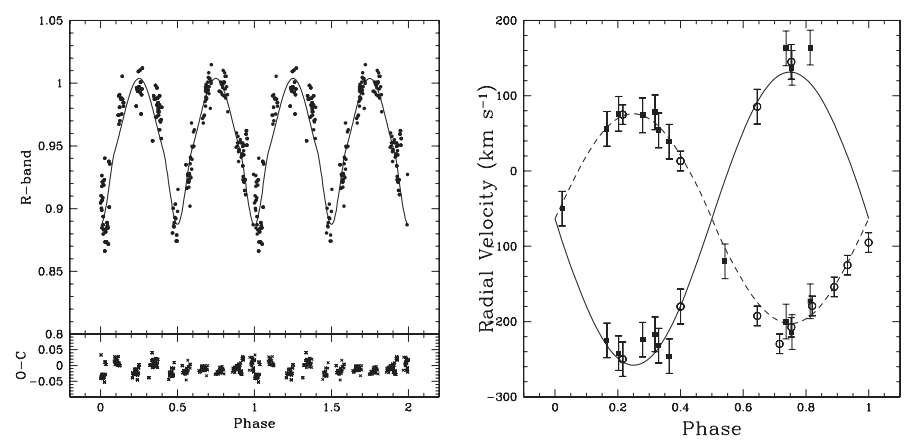

Figure 1: Phased $R$ light curve and radial velocity curve of $\mathrm{Wd} 13$, respectively. Radial velocity points from Ritchie et al. (2010) are also included (black squares).

The physical and orbital parameters of the members of the four systems (period, masses, radii, surface gravities of the components, eccentricity, inclination etc.), resulted from modelling light and radial velocity curves using PHOEBE software (Prsa \& Zwitter 2005). Our final results will be presented in Koumpia \& Bonanos (in prep.).

Wd13: is a semi-detached, double-lined spectroscopic binary (B0.5Ia ${ }^{+} /$WNVL and O9.5B0.5I types; Ritchie et al. 2010) in a circular orbit (see Fig. 1). Its spectra show both absorption and emission lines. It provides the first dynamical constraint on the mass of the magnetar progenitor and has important implications for the threshold mass which gives rise to black holes versus neutron stars.

Wd36: is an overcontact, double-lined spectroscopic binary system (OB-type) in a circular orbit.

WR770: is probably a double-contact binary system in an almost circular orbit. It is a single-lined spectroscopic binary. The spectroscopic visible star is a Wolf-Rayet star of spectral type WN6-7 (Negueruela \& Clark 2005), with line widths of $2000 \mathrm{~km} \mathrm{~s}^{-1}$.

Wddeb: is a double-lined spectroscopic binary system (OB-type) with an eccentricity of almost 0.2. Being a detached system, it contains the first main-sequence stars detected in the cluster, yielding masses for two of the most massive unevolved stars in Wd1.

\section{Conclusions}

Having obtained the physical and orbital parameters of each system, we confirm the high mass of Wd13 and therefore the high mass of the magnetar progenitor, also found by the independent study of Ritchie et al. (2010). We also compared our results with stellar models of evolution of single stars (Claret 2004).

Acknowledgements: We acknowledge research and travel support from the IAU and the European Commission Framework Program Seven under the Marie Curie International Reintegration Grant PIRG04-GA-2008-239335. IRAF is distributed by the NOAO, which are operated by AURA, under cooperative agreement with the NSF.

\section{References}

Bonanos, A. Z. 2007, AJ, 133, 2696

Claret, A. 2004, $A \& A$ A, 428, 1001

Lanz, T. \& Hubeny, I. 2003, ApJS, 146, 417

Muno, M. P., Clark, J. S., \& Crowther, P. A. et al., 2006, ApJ, 636, 41

Negueruela, I. \& Clark, J. S. 2005, A\&A, 436, 541

Negueruela, I., Clark, J. S., \& Ritchie, B. W. 2010, A\&A, 516, 78

Prsa, A. \& Zwitter, T. 2005, ApJ, 628, 426

Westerlund, B. 1961, AJ, 66, 57 